\title{
Reproduction et révolution normative : mariage, monogamie et biologie sous le III $^{\mathrm{e}}$ Reich
}

Johann Chapoutot

\section{(2) OpenEdition}

\section{Journals}

Édition électronique

URL : http://journals.openedition.org/ifha/491

DOI : 10.4000/ifha.491

ISSN : 2198-8943

\section{Éditeur}

IFRA - Institut franco-allemand (sciences historiques et sociales)

\section{Édition imprimée}

Date de publication : 30 septembre 2012

Pagination : 261-289

ISSN : 2190-0078

\section{Référence électronique}

Johann Chapoutot, «Reproduction et révolution normative : mariage, monogamie et biologie sous le IIIe Reich », Revue de l'IFHA [En ligne], 4 | 2012, mis en ligne le 14 février 2013, consulté le 20 avril 2019. URL : http://journals.openedition.org/ifha/491 ; DOI : 10.4000/ifha.491

Ce document a été généré automatiquement le 20 avril 2019

(CIFHA 


\title{
Reproduction et révolution normative: mariage, monogamie et biologie sous le III Reich
}

\author{
Johann Chapoutot
}

1 Tout comme la France des Croix de bois, l'Allemagne a eu ses cauchemars oliganthropiques : la pire conséquence de la Grande Guerre n'est, pour de larges cercles conservateurs et nationalistes allemands, pas la défaite, ni même le traité de Versailles, mais la saignée démographique de quatre à cinq ans de combats, de blocus, de famine, puis de grippe espagnole. Une guerre est une confrontation que l'on peut rejouer, un traité peut être renégocié. La structure d'une pyramide des âges est au contraire affectée d'une inertie sur laquelle la nervosité de la décision politique ou militaire n'a pas de prise : le véritable passif des Flandres, de la Somme et des tranchées est bel et bien là.

2 L'effroi devant le dépeuplement et la dénatalité n'est pas un apanage allemand : la France vote une législation anti-avortement sévère en 1920, et ne cessera de chercher, d'HBM en lois sociales, la martingale nataliste, jusqu'au code de la famille de 1939.

\section{Une extinction biologique du peuple allemand?}

3 En Allemagne, la perception de l'hémorragie est non seulement aggravée par la défaite, mais elle revêt de surcroît, dans un pays bouleversé par des mutations sociales et culturelles massives et rapides, un aspect apocalyptique particulier : l'Allemagne vit une quintuple fin $\mathrm{du}$ monde (militaire, politique, diplomatique, puis financière et économique) dont l'anémie démographique semble être l'expression biologique la plus intense et la plus tragique. Pendant que les lecteurs font un triomphe à Oswald Spengler, professeur de biologie dans un lycée de Hambourg articles, brochures et essais se multiplient sur l'aspect démographique de la catastrophe allemande de 1914-1919 ou de 1914-1923. 
4 Très classiquement, tout ce qui définit le mode de vie urbain moderne y est cloué au pilori : l'émancipation des femmes, le célibat, la réduction du nombre d'enfants par foyer, la contraception, mais aussi cette homosexualité qui, grâce aux films et publications de médecins militants comme Magnus Hirschfeld, s'affiche comme une autre normalité parfaitement légitime.

5 L'extrême-droite völkisch, celle-là même qui prétend mettre le Volk comme organisme biologique au centre de ses préoccupations, participe de cette angoisse nataliste et démographique. Réfutant le pessimisme méthodologique d'un Spengler pour qui, semblet-il, la sénescence et la finitude d'un peuple sont des phénomènes nécessaires et fatals, les nazis développent un discours à la fois plus angoissé (et anxiogène) et plus volontariste la tonitruance de la volonté étant d'autant plus bruyante que le constat démographique est plus noir.

6 Dans une brochure intitulée Peuple en péril et parue en $1934^{1}$, l'un des deux éditeurs attitrés des publications nazies (Lehmann, à Munich) livre au public un constat proprement apocalyptique : la catastrophe démographique y est ici surdéterminée par un sentiment obsidional très vif, qui présente une Allemagne moribonde, encerclée par un monde en pleine santé, et minée de l'intérieur par la croissance exponentielle des dégénérés, des asociaux et des ratés. Toutes les ressources de l'illustration graphique sont ici mobilisées, du diagramme implacable à l'infographie effrayante, qui fait démesurément grossir un visage slave patibulaire à côté d'une belle tête aryenne qui, elle, disparaît de la feuille : 59 millions de Germains et 65 millions de Slaves en Europe en 1810 - les projections pour 1960 sont terrifiantes : 160 millions de nordiques seulement contre 303 millions de Slaves ${ }^{2}$. Une autre illustration montre la Pariser Platz, devant la porte de Brandebourg, se vider peu à peu : 4 millions d'habitants à Berlin en 1925, 100000 en $2075^{3}$.

7 Le commentaire souligne ces messages : "Nous sommes un peuple agonisant ${ }^{4}$ ", vidé de sa substance par l'absence de renouvellement démographique, et par la croissance des populations de malades, de criminels, d'allogènes et, à l'extérieur, de Slaves.

8 Dans une postface très ferme, Arthur Gütt répète ce constat et vitupère des normes morales qu'il rend responsables d'une dégradation ontologique inédite du peuple allemand : la « loi de la nature » élimine les faibles et les ratés, et commande que « soit maintenu en vie le peuple allemand ${ }^{5}$ ", coûte que coûte. Une morale dévoyée a commandé de soigner les dégénérés et de tolérer l'absence d'enfants: la "sélection naturelle » a été neutralisée par cette « idéologie du dernier millénaire, qui nous a imposé l'impératif moral d'assister tout ce qui est malade et faible ${ }^{6}$ ».

9 C'est tout le contraire que commande une éthique dérivée des lois de la nature, qui crée des « devoirs envers la famille allemande, envers le peuple allemand et envers l'avenir de l'Allemagne ${ }^{7}$ ». Les personnes âgées, dès lors, «n'ont de droit à l'assistance » que si et seulement si « ils ont contribué à la fertilité allemande » et si ces vieux ont su « donner au peuple allemand une éternelle jeunesse ${ }^{8} »$.

10 On le voit, Arthur Gütt défend que «tout doit être subordonné à cette fin de notre politique raciale», tout, "y compris les impératifs qui régissent notre sexualité et nos familles » et qui ne doivent plus participer de ces "doctrines dépassées et fausses ${ }^{9}$ » léguées par le christianisme.

11 De 1936 à 1945, le même éditeur, Lehmann, publie une série de monographies intitulée «Biologie politique - Cahiers pour une science et une politique biologique», dont les 
titres renseignent sur « La guerre des naissances », appellent à « La guerre contre la mort infantile ", ou décrivent des «Peuples au bord de l'abîme ${ }^{10}$ ".

L'argumentation est, à chaque fois, identique, et reprend tout ce dont la brochure de 1934 était déjà un compendium.

L'originalité de cette abondante littérature démographique nazie est double. Elle touche d'abord à toute l'histoire: de même que le concept de race et la lecture raciste de l'histoire permettent d'éclairer le réel contemporain et la totalité du devenir humain, de même cette angoisse de l'oliganthropie est-elle projetée sur la totalité des cas d'espèce historique. Si les civilisations grecque et romaine (nordiques) ont disparu, par exemple, c'est en grande partie pour des raisons d'effondrement démographique, dû à l'hémorragie de guerres fratricides et à une dénatalité causée par la décadence des mœurs ${ }^{11}$. De même, la guerre de Trente ans révèle-t-elle l'hostilité d'un monde coalisé contre l'Allemagne et conspirant à sa disparition biologique, une lecture que les nazis estiment confirmée par la Grande Guerre.

Cette étiologie de la mort des peuples, et le constat de l'urgence contemporaine, conduit à la formulation d'un catalogue de mesures très classiques et attendues.

De l'encouragement bruyant et très conventionnel à l'accroissement numérique de la famille allemande à la lutte classique contre l'avortement, l'homosexualité et l'emploi des femmes (sauf en temps de guerre...), la panoplie classique du dispositif nataliste peuple les argumentaires, puis les dispositions réglementaires et législatives. Mesures incitatives (allocations...) et sanctions (fiscales et pénales) frappant l'absence de fécondité sont bien connues et se retrouvent ailleurs.

Le discours nazi développe cependant une radicalité critique et programmatique peu commune : il s'agit, à le lire, de détruire les fondements d'une culture, d'une normativité nocive et néfaste pour instaurer une politique biologique, une "naturgesetzliche Politik ", c'est-à-dire, littéralement, une politique qui respecte la nature comme seule loi et qui ait la nature pour seule loi, i.e. pour seul principe et pour seule fin.

La Naturgesetzlichkeit - le respect de la nature comme seule instance législatrice caractérise à bien des égards, sinon essentiellement, la totalité du discours normatif nazi. Pour ce qui nous occupe ici, nous constatons que, parallèlement à l'arsenal de mesures que nous évoquions, s'ajoute un assaut singulier contre l'institution même du mariage, non seulement dans ses conséquences juridiques, mais également dans son principe même. Cet assaut se déroule en trois vagues successives, qui visent, dans un ordre chronologique - qui correspond également à une gradation logique : le statut des enfants illégitimes (hors mariage), les conditions d'exercice du droit de divorce puis, enfin, la norme monogamique elle-même.

\section{Enfant naturel, enfant de la nature ${ }^{12}$}

Le premier des trois fronts ouverts contre le mariage concerne les conséquences juridiques de cette institution sociale : la différence de statut entre les enfants issus d'une union sanctionnée par le mariage et ceux qui sont nés hors-mariage. Le code civil allemand de 1900, le Bürgerliches Gesetzbuch, consacre en effet l'inégalité légale entre les enfants légitimes (eheliche Kinder : littéralement, enfants issus d'un mariage) et les enfants illégitimes (uneheliche Kinder, littéralement: enfants hors-mariage). La lutte contre cette 
inégalité de statut est, aux yeux des nazis, une question de principe autant que de pure comptabilité démographique.

Du point de vue démographique, pour commencer, il est inconcevable de flétrir des fillesmères qui apportent des enfants à l'Allemagne. Si la société et la culture chrétienne condamnent cette reproduction hors-mariage, c'est par oubli des nécessités naturelles de la préservation de la race ou, pire, par hostilité à celle-ci.

Théoricien longtemps apprécié de Himmler, Richard Walther Darré soulève la question de principe, et déclare la guerre à une moralité sociale veule et inepte :

«Si l'on considère la question de l'enfant illégitime du point de vue de l'amélioration raciale, le problème est tout différent, car c'est la valeur héréditaire qui prime, c'est-àdire la provenance raciale - et dans un deuxième temps seulement la question de la conception dans ou en dehors du mariage ${ }^{13}$ ». Dix ans plus tard, Darré revient sur cette question en déplorant que, dans l'Allemagne nazie, « on se demande encore trop souvent dans quelles conditions matrimoniales un enfant est né au lieu de s'intéresser à la valeur raciale de l'enfant ». Le mariage, chez les Germains, avait selon Darré pour principe et pour fin le développement de la vie de la race, et non l'épanouissement de deux individus. Aux yeux de Richard Darré, c'est le Code civil de 1900 et «l'idéologie libérale qui a renversé la hiérarchie des valeurs (umgewertet) ». Contre cette première Umwertung, cette première inversion des valeurs, il convient de remettre les choses à l'endroit. La seconde Umwertung sera donc une révolution qui permettra de revenir aux valeurs essentielles de la race : ce qui importe avant tout, c'est que des enfants naissent et que le «mariage » soit remis «au service de la reproduction des générations ${ }^{14}$ ». Pour trancher les débats contemporains, Darré propose un critère sûr, un critère de responsabilité biologique: "Le concept d'un enfant dont on serait responsable devant nos ancêtres donne un critère sûr pour formuler une position claire dans le brouhaha qui règne autour de l'enfant illégitime. Il permet également de poser les fondations d'une moralité allemande conforme à la race et responsable devant elle $^{15} »$. Ahnenverantwortung: l'acte de procréation ne peut comparaître que devant le tribunal de la race, et non d'une quelconque Église ou d'une morale bourgeoise veule et philistine.

Le droit germanique prend donc la race pour principe et pour fin. Droit pleinement naturel, en ce sens qu'il transcrit les commandements de la nature et sert ses fins, il s'oppose à toute anti-nature: celle du christianisme, du droit civil et des préjugés bourgeois. Dans les colonnes de la revue Deutsches Recht, un juriste s'intéresse ainsi au « concept de Sippe [famille, tribu] dans le droit germanique ». Il rappelle ainsi que, au rebours de l'article 1589 du BGB allemand qui ne reconnaît à l'enfant naturel aucun lien avec la famille du père, « la loi norvégienne du 10 avril 1915 a rétabli dans ses droits les conceptions sociales des anciens Germains dans la mesure où elle a décidé que „l'enfant illégitime jouit [...] du même statut juridique par rapport au père que par rapport à la mère ${ }^{{ }^{\prime 16}}$ ». Les Norvégiens ont su renouer avec les normes de « ces ancêtres [qui] avaient une pensée juridique plus juste et plus conforme à la biologie ${ }^{17}$ ». Le droit civil norvégien est conforme à la tradition germanique, mais aussi aux acquis les plus récents de la science de la race : « la justification de cette norme est que le lien de sang naturel avec le père et la mère est identique, et qu'il transmet la même hérédité à l'enfant, de telle sorte que la science de l'hérédité nous commande de conclure juridiquement à la parenté substantielle et biologique immédiate de l'enfant et des deux parents ${ }^{18}$ ». Le droit civil, comme toute norme juridique, n'est légitime qu'en tant qu'il est induit, ou traduit, des 
lois de la nature, le droit positif se bornant à être une translittération des normes naturelles.

Ce qui se joue dans ce débat autour de l'enfant illégitime et de son statut n'est rien moins que la valeur du mariage comme institution sociale. Que le mariage soit affecté d'une valeur sacrale par des Églises ne peut entrer en ligne de compte : ce qui importe est la valeur biologique de cet acte juridique. Le droit ne peut et ne doit pas entrer en contradiction avec la nature. S'il l'entrave, il doit être réformé. Comme l'écrit un journaliste du journal de la SS, Le Corps noir :

24 «Le mariage est un instrument dont se sert la nature pour projeter la race dans l'éternité. La nature se moque du droit, elle veut [...] un acte biologique. Tout le reste n'est qu'œuvre humaine, certes nécessaire, mais artificielle. Quiconque s'interroge sur la signification profonde du mariage ne peut donc jamais partir que de l'essence et de la volonté de la nature ${ }^{19} \%$.

Cette conception du droit, de surcroit sur un sujet aussi sensible que les mœurs sexuelles et la morale bourgeoise, ne va pas de soi, et les Églises s'offusquent des velléités nazies de donner à l'enfant illégitime une position équivalente à l'enfant d'un couple marié. Dans un article publié dans les colonnes de la revue Deutsches Recht, le docteur en droit et Untersturmführer Kurt Schmidt-Klevenow, membre de l'Office central de la race et de la colonisation de la SS (RuSHA), évoque les «réserves de nos ennemis idéologiques, en particulier des Églises », tout en précisant que « le national-socialisme n'est pas disposé à faire des compromis avec telle ou telle proposition dogmatique libérale-bourgeoise ou cléricale-dogmatique ». Le nazisme, qui est une « raciologie appliquée » vise à " intégrer l'enfant illégitime dans la communauté raciale en le tirant une bonne fois pour toutes du marais de la culture libérale-bourgeoise ou cléricale. La prétendue morale bourgeoise ou religieuse ont généralement fait de l'enfant illégitime un être de seconde catégorie pour la simple raison qu'il était né hors-mariage ${ }^{20} »$.

26 En bonne orthodoxie nazie, l'auteur précise que «tout droit tout être fondé biologiquement sur le sang, sur la foi en une législation éternelle de la nature. Plus un droit se rapproche de l'ordre naturel, meilleur il est. C'est seulement ainsi qu'il peut remplir son rôle et illustrer les lois sacrées de la nature et de la vie ${ }^{21}$ ».

Le front nazi est donc double : il s'agit à la fois de combattre les préjugés constitutifs d'une morale victorienne aveugle et bête, mais aussi d'affronter cette culture chrétienne dont les normes bourgeoises sont dérivées.

Révérence obligée, et concession rhétoriquement utile, l'auteur ne peut se dispenser d'un hommage appuyé à la famille allemande, que, parbleu, le national-socialisme ne vise pas à détruire. C'est que certains s'en inquiètent, quelques voix divergentes qui font part de leurs doutes et réticences jusque dans les colonnes des publications nazies. Ainsi de Friedrich Lenz qui, dans les colonnes de Volk und Rasse $e^{22}$, vitupère les «enfants illégitimes » et toute menace de dissolution de la famille, notamment le concept de " mariage biologique ${ }^{23}$ " forgé par certains collègues. Cet article provoque la colère d'Heinrich Himmler qui interdit à son auteur toute publication ultérieure du même acabit ${ }^{24}$.

29 C'est que le Reichsführer SS prend des initiatives auxquelles Schmidt-Klevenow rend hommage : « La conséquence logique [de cette conception du droit] est la nécessité d'une sélection et notre devoir de donner aux enfants illégitimes sains et de valeur de meilleures conditions de vie. C'est dans cette mesure que le Reichsführer SS a bien mérité 
des familles et des enfants en créant le Lebensborn », institution dictée «par la nécessité ${ }^{25}$ » naturelle.

Militant de la « réévaluation des valeurs » héritées du christianisme, le Reichsführer SS Heinrich Himmler a en effet créé une institution inédite en 1936 : l'association « Fontaine de vie » (Lebensborn e. V.) qui doit accueillir dans ses hospices toutes les filles-mères de bonne race, et leur offrir tout soutien pour accoucher, puis pour élever leurs enfants dans les meilleures conditions ${ }^{26}$. A ceux qui, du côté des clergés protestant et catholique notamment, ou des chaisières de tout poil, froncent les sourcils ou tordent le nez, Himmler rétorque qu'au sein du Lebensborn, "c'est un amour du prochain concret que nous pratiquons. C'est ce que devraient comprendre Messieurs les Pasteurs et les curés qui tonnent, du haut de leurs chaires, contre les filles-mères et qui ne savent pas le mal qu'ils infligent à ces pauvres femmes en montant contre elles toute la société27 ».

31 Cet "amour du prochain concret" relève d'une moralité supérieure, qui transcende et invalide la moralité chrétienne, si hostile à la reproduction de la race : "concret», il s'oppose à des principes abstraits, désincarnés et désincarnants, pour permettre la reproduction et la production de substance biologique de bonne race.

On voit que, sur la question de l'enfant illégitime, une conception radicalement autre du droit s'expose : le droit doit être selon les nazis la pure et simple transcription du fait de nature ; le droit allemand doit être révolutionnaire et révolutionné, i.e. revenir au point de départ germanique, à la proximité germanique à la nature : Darré oppose à la première « inversion des valeurs » (Umwertung) un second renversement, qui permettra de rendre à la norme la légitimité en faisant d'elle, à nouveau, la translittération des décrets de la nature.

\section{Pour la dissolution de l'union stérile}

Le second combat des idéologues, raciologues et juristes nazis vise les conditions de dissolution du mariage. Si, comme le dit le Corps noir, la seule fin du mariage est la procréation de nombreux enfants, il est opportun d'assouplir la législation du divorce pour permettre une séparation des couples infertiles. Cet assouplissement est obtenu par la loi de 1937 portant réforme du mariage et fait l'objet d'une justification biologique. Comme l'explique Franz Wieacker, professeur à Kiel, dans les colonnes de Deutsches Recht, « la nouvelle vision du monde confère à nouveau à la famille et au mariage le caractère d'un service ${ }^{28}$, d'une fonction au sein d'un ensemble qui les dépasse ${ }^{29} »$. Il ne s'agit certes pas, en réfléchissant à une réforme du droit matrimonial, de bouleverser cette belle et noble institution, en autorisant le divorce ad libitum. Mais, a contrario, il doit être mis fin aux limitations cléricales ou aux héritages religieux: "Il n'y aura pas de divorce arbitraire, mais aucune institution matrimoniale transcendante ne doit empêcher la dissolution de mariages malades au profit de la communauté du peuple ${ }^{30} »$. Son collègue Karl Larenz, professeur à Kiel, le rejoint: "Le législateur doit avoir le courage de reconnaître qu'un mariage désespérément abîmé n'est en vérité plus un mariage [...]. Un lien, qui a cessé d'en être un, devrait être déclaré formellement rompu ${ }^{31}{ }$ - ici encore, le droit ne semble devoir qu'acter un fait. Il en va des mariages où l'amour et la confiance ne règnent plus et où, avant tout, la procréation d'enfants est devenue impossible.

Le juriste Karl Mössmer prônait, dans les débats préparatoires à la réforme du mariage, pour une intégration du « concept de communauté » à toute réflexion sur «mariage et 
divorce $^{32}$ ». La communauté, l'auteur ne l'écrit pas ici mais il vaut de le rappeler, n'est ni la société, ni une civitas transcendante gagée sur le sacré. Dans une société - groupe humain fondé sur la libre contractualisation des individus -, un mariage librement concluable et dissoluble est pensable. A l'inverse, dans une civitas religieuse, constituée par des postulats et des catégories forgées par l'Église, le mariage est un sacrement aussi indissoluble et éternel que l'est le lien entre le Créateur et ses créatures. La communauté ( Gemeinschaft) nazie n'est rien de tout cela: ni libre (au sens rousseauiste et révolutionnaire), ni (faussement) sacrée, elle est organique. Organisme biologique, elle est fondée sur la mutuelle participation de tous à la même substance et a pour fin la reproduction de cette substance par la procréation d'enfants.

Dès lors, le mariage ne peut être conçu que comme "une communauté de vie ( Lebensgemeinschaft) entre deux personnes saines de même race et de sexe différent à toute fin de préservation et d'encouragement du bien commun par une cohabitation harmonieuse qui vise à produire des enfants sains de bonne race ainsi qu'à les éduquer pour qu'ils deviennent des membres valables de la communauté du peuple ${ }^{33} »$. Cette définition s'éloigne on ne peut plus de la conception libérale du mariage esquissé par Kant, puis consacrée par le BGB de 1900 : le mariage était, dans les termes de Kant, un contrat régissant l'usage des parties génitales de chacun et, dans les termes du droit civil, un acte synallagmatique révocable à loisir. Il est ici moyen en vue d'une fin : procréation et production d'une saine substance biologique.

En amont, la prévention prophylactique de mariages indésirables est donc nécessaire, comme le disposent les lois de 1933 et de 1935 qui interdisent l'union et la reproduction d'éléments sains avec des éléments à l'hérédité gâtée (lois du 14 juillet et du 24 novembre 1933) ou avec des juifs (lois de Nuremberg). En aval, le débat - souhaitable - sur le divorce et son assouplissement reste parasité par un tabou et une erreur. Le tabou est celui qui est imposé par le "droit du mariage catholique", qui y voit un sacrement indissoluble. L'erreur, a contrario, est celle du " libéralisme », qui trouve son accomplissement dans « la législation du mariage soviétique »: le mariage ne serait qu'une "affaire de droit privé » qui ne serait soumise à "aucune autre condition de dissolution que la simple dénonciation unilatérale ${ }^{34}$ ». Cette conception «libérale-soviétique» (sic) est inacceptable : les époux doivent être conscients qu'ils sont soumis à un devoir (envers la communauté, envers la race) qui les dépasse. Cependant, et pour les mêmes raisons, un mariage qui ne sert plus la communauté raciale doit pouvoir être dissout, sans que ne pèsent plus sur le divorce les préventions, préjugés et interdits hérités d'une conception cléricale et sacramentelle.

L'actuelle « limitation rigide des causes de divorce à la seule faute constatée ${ }^{35}$ » empêche des époux convaincus de la non-viabilité de leur union de divorcer et les conduit à contourner la loi en mentant devant le juge pour que celui-ci accorde un divorce pour faute. Il serait préférable d'assouplir cette législation pour que des époux puissent divorcer, « en particulier quand leur mariage est resté stérile ${ }^{36}$ ». Cet assouplissement ne sera en aucun cas la porte ouverte à des déflagrations familiales en série, car les juges veilleront: il va de soi que «des mariages qui offriront encore une quelconque perspective de reproduction féconde devront être maintenus, au contraire des unions qui, irrémédiablement détruites, devront être dissoutes ${ }^{37}$ ».

38 Face à de tels arguments, si puissamment fondés sur une conception biologique de la communauté politique et sur la finalité reproductive de toute union, les hésitants et les réticents ont peu de choses à opposer : les Églises défendent le dogme, mais celui-ci est 
répudié sur le fondement de son anti-naturalité, voire de son hostilité à la race et à son développement. Quant aux conservateurs de tout poil, ils ne peuvent opposer à la déferlante des raisonnements biologiques et à l'apodicticité de la nécessité naturelle qu'une litanie répétitive et des arguments tautologiques. Le discours nazi, aux termes duquel les institutions sociales doivent servir les finalités de la nature, se caractérise par une radicalité qui bouscule les vieilles lunes bourgeoises et les préjugés sociaux hérités qui n'ont à opposer qu'une plainte vaine et non argumentée selon laquelle il faut défendre la famille traditionnelle parce qu'il en a toujours été ainsi.

La conception nazie suscite des résistances, mais parvient à s'imposer, non seulement dans la lettre des normes juridiques, mais aussi dans la réalité des pratiques sociales, comme en témoigne le succès des Lebensborn.

On constate que, alors que le combat pour l'égalité de statut de l'enfant illégitime contestait au fond l'obligation d'une reproduction sexuée dans le cadre d'une union sanctionnée par la loi, ce débat pour l'assouplissement des conditions du divorce constitue un second front qui vient dévaluer le mariage monogamique et unique comme cadre et lieu impératif, ou même principal, de la reproduction. La voie était donc ouverte à la contestation du principe monogamique lui-même.

\section{Abolir la monogamie?}

41 Dans le Völkischer Beobachter des 24-26 décembre 1939, Rudolf Hess, «représentant du Führer " publie une lettre ouverte supposément adressée à une jeune femme enceinte, fiancée à un homme mobilisé dans la Wehrmacht et récemment tombé sur le front de Pologne. Fiancée et non mariée, cette femme doit donc donner naissance à un enfant illégitime, ce qui la désespère et la conduit à confier son désarroi à Hess.

42 Cette démarche supposée de la fiancée d'un héros, d'une jeune femme dont la faute est éclipsée par le sacrifice du père de son enfant, permet aux éléments d'avant-garde de la hiérarchie nazie d'avancer une idée qui, dans le contexte d'une guerre qui vient de débuter, tend à se banaliser, après avoir été l'apanage des seuls éléments les plus en pointe du racisme le plus conséquent et le plus "révolutionnaire» au sein de la SS. L'article signé par Hess est remarquablement construit: le "représentant du Führer » commence par exprimer sa profonde sympathie pour une jeune femme dont il se propose de reconnaître l'enfant en en devenant le "parrain ${ }^{38}$ ». Il va de soi, selon Hess, qu'en bonne solidarité nationale-socialiste, «vous et votre enfant serez traités et aidés de la même manière que si le mariage avait déjà été conclu ${ }^{39} »-$ une fois encore, le fait de nature (la conception) vaut acte juridique.

$43 \mathrm{Au}$ fond, Hess aurait pu en rester là : la faute du couple ayant été rachetée par la mort du père, cette émouvante histoire aurait pu raffermir le principe même du mariage. Or, il n'est, dans cet article, aucunement question de faute, bien au contraire.

$\mathrm{Si}$, précaution rhétorique, «le mouvement national-socialiste voit dans la famille la cellule fondamentale du peuple », il reste que « dans des moments exceptionnels [...] des mesures particulières divergentes par rapport aux règles d'usage doivent être prises, particulièrement en temps de guerre, où meurent tant de jeunes hommes, et où toute nouvelle vie revêt une importance particulière pour la nation ».

L'état d'exception créé par la guerre suspend donc les "vieux usages vénérables » et efface les «frontières des us et habitudes bourgeois, qui sont sans doute nécessaires » en 
temps normal. Les adjectifs "vénérable» et "bourgeois » ne sont pas précisément mélioratifs sous la plume de Hess, comme de tout chef nazi... Expression d'un vieux monde étriqué et exsangue, les règles d'hier sont donc suspendues par la guerre, mais pas seulement : elles sont condamnées sur le fondement de leur obsolescence. Que cet effort d'émancipation des normes héritées n'ait rien d'aisé est concédé par Hess qui, fort habilement, prétend se mettre à la place de la jeune femme et du lecteur de cette lettre ouverte : «Croyez-moi, cela ne m'a pas été facile non plus de me libérer de traditions qui avaient toujours été évidentes pour moi. Mais, en tant que national-socialiste, je peux vous dire ceci: la loi suprême, en temps de guerre comme en temps de paix est la préservation de la race. Toutes les autres lois, us et conceptions doivent se soumettre à cette loi supérieure ».

Hess écrit bien: "En temps de guerre comme en temps de paix »... est-ce à dire que les jours du mariage sont comptés? En tant que norme, en tant que condition obligatoire d'une reproduction sexuée légitime, indubitablement: «En temps de guerre, le fait de donner la mort revêt une signification nouvelle, car il sert la préservation de la nation. Notre rapport aux mères-filles et aux enfants nés hors-mariage doit connaître une évolution semblable, et pour les mêmes raisons, en temps de guerre comme en temps de paix. A quoi servirait-il qu'un peuple gagne s'il devait périr à cause des sacrifices consentis pour la victoire?».

Si l'on a appris à tuer pour défendre la nation en s'affranchissant des interdits hérités qui frappent usuellement le meurtre d'autrui, il faut aussi apprendre à enfanter en dehors des cadres normatifs légués par un passé dépassé. La guerre ici ne compte plus: Hess parle d'une révolution normative définitive qui prend en compte le fait que « le bien de la communauté, la vie de la race est située plus haut que tous les principes imaginés par les hommes, plus haut que toutes les normes morales qui, pour être l'expression d'une coutume, n'en sont pas pour autant la morale en soi, plus haut, enfin, que tous les préjugés ».

us sociaux - et les institutions juridiques qui en sont les gardiennes - sont donc dégradés au rang de simple " coutume », inférieure à la «morale en soi », c'est-à-dire cet ensemble de normes qui ont pour fin la vie et le développement de la race.

La chronologie nous révèle que cet article de Rudolf Hess, publié fin décembre 1939, intervient opportunément deux mois après une initiative du Reichsführer SS, Heinrich Himmler, qui avait provoqué des commentaires outrés et des réactions hostiles dans le milieu, conservateur, des officiers de la Wehrmacht.

Ce n'est pas la première fois qu'Himmler intervient dans le domaine de la conjugalité de ses hommes : dès le 31 décembre 1931, il avait soumis tout mariage d'un membre de la SS à l'approbation de l'office central de la race et de la colonisation (RuSHA) sur le fondement de l'appartenance raciale de la promise. Rien que de très logique : comme la SS se voulait l'élite raciale du Reich à venir, il était impératif que la sélection raciale la plus stricte gouvernât son recrutement ainsi que le mariage de ses hommes.

51 Le 28 octobre 1939, Himmler édicte un ordre qui n'a pas pour objet la qualité du "matériel humain » SS, mais bien sa quantité. Le Zeugungsbefehl (ordre de reproduction) anticipe sur les pertes humaines de la guerre en cours et à venir, et ordonne aux soldats de la Waffen-SS d'engrosser au plus vite leurs épouses, car «le plus beau cadeau d'une veuve de guerre est toujours l'enfant de l'homme qu'elle a aimé ». Quant à ceux qui ne 
sont pas encore mariés, ils reçoivent également l'ordre de féconder toute femme allemande disponible :

"Au-delà des limites imposées par des lois et des usages bourgeois par ailleurs peut-être nécessaires, le fait de devenir les mères de soldats partant au front va devenir un devoir supérieur, y compris en dehors du mariage, pour des jeunes femmes et filles de bon sang, non pas par légèreté, mais pour les raisons morales les plus sérieuses et les plus profondes ». C'est par devoir que l'on combat et que l'on tue. C'est tout autant par devoir que l'on copule et procrée.

Ce «devoir sacré » est dicté par la mort qui frappe déjà, mais aussi par les tâches de colonisation qui attendent la race germanique-nordique, sur des espaces d'une ampleur inédite. Les enfants issus de cette sexualité militante et militaire bénéficieront d'une tutelle « de représentants du Reichsführer SS » et jouiront de tous les moyens matériels nécessaires à leur éducation "jusqu'à leur majorité", dans le cadre des hospices du Lebensborn, précise Himmler dans une circulaire complémentaire à la SS et à la police allemande du 30 janvier 1940. Dans ce second texte, Himmler fait état des «malentendus » provoqués par son ordre du 28 octobre 1939: il ne s'agit pas, pour les hommes de la police et de la SS, de «s'approcher de la femme d'un camarade » déjà parti au combat! L'honneur militaire et le simple bon sens le défendent, tout comme ils commandent de mobiliser toute femme disponible dans ce combat des naissances, "question vitale pour notre peuple ». Le Reichsführer SS, que l'on savait peu sensible à l'humour leste, rappelle une nouvelle fois qu'il s'agit, pour les femmes et les hommes non mariés, d'un devoir « sacré, supérieur à toute légèreté et à toute plaisanterie ».

Quand il rédige ses lignes pour légitimer la pratique d'une sexualité non pas seulement préconjugale, mais clairement extraconjugale, Himmler peut se fonder sur une idée déjà répandue et acceptée dans le cercle le plus élevé de la hiérarchie nazie: enfanter, de quelque manière que ce soit, est le devoir de toute femme. Mieux : il s'agit de l'exact équivalent, pour la femme, du devoir militaire qui incombe aux hommes. Comme le déclare Hitler aux femmes allemandes lors d'un discours de 1934 à Nuremberg :

«Tout ce que l'homme consent comme sacrifices dans la lutte que mène son peuple, la femme le consent dans son combat pour la préservation des familles de son peuple. Le courage que l'homme démontre sur le champ de bataille, la femme le démontre aussi, par son dévouement patient constant, par son amour patient et par ce qu'elle endure. Chaque enfant qu'elle met au monde est une bataille qu'elle gagne pour l'existence de son peuple, contre le néant qui menace ${ }^{40}$ ». Ce qui est, en temps de paix, conçu et prononcé comme une analogie méliorative censée valoriser le grand renfermement des femmes dans l'espace privé, est à entendre au sens le plus littéral en temps de guerre: les femmes combattent avec la seule arme dont leur faible nature dispose, i.e. leur ventre.

Hitler, lui-même célibataire jusqu'au jour de son suicide, approuve en privé toutes les entorses constatées dans les rangs de la SS à l'obligation d'une sexualité monogamique et conjugale. S'il réagit avec vigueur quand Goebbels délaisse son épouse pour une actrice tchèque, car le scandale dessert le régime et blesse la mère prolifique de cinq enfants allemands qu'est Magda Goebbels, Hitler «se réjouit que justement une troupe d'élite comme la Leibstandarte considère son devoir de mettre des enfants au monde comme un devoir de race ». Ces propos du Führer font suite à un satisfecit qu'Himmler vient de se décerner : «Vu les pertes que la SS a dû subir dans les rangs de ses jeunes hommes encore célibataires, je suis content d'avoir donné cet ordre [de reproduction]. De cette manière, le sang de ces hommes est au moins conservé dans un enfant ». Encouragé par l'ambiance 
qui règne à table, un convive de la Wolfschanze raconte une plaisanterie qui court sur la signification de trois des nombreux acronymes qui, depuis 1933, se multiplient dans le Reich: «Savez-vous ce que veut dire BDM, WHW et NSV ${ }^{41}$ ? Bin Deutsche Mutter, Wie Hitler Will. Nun Suche Vater (je suis une mère allemande, comme le veut Hitler. Maintenant, je cherche un père !) ${ }^{42}$ ». Le sténographe note que le Führer rit avec plaisir à ce trait d'humour, de conserve avec les autres commensaux.

Le mariage et l'ensemble des normes et des représentations qui l'entourent indisposent visiblement Hitler, qui s'offusque de la médiocrité bourgeoise dont elle est le fondement et la source: s'emportant contre les "tartuffes ${ }^{43}$ ", Hitler vitupère cette "morale mensongère ${ }^{44}$ » qui conduit des officiers à quitter l'armée quand ils ont eu des relations extraconjugales ${ }^{45}$. Rien de tout cela ne lui semble ni très sain, ni très naturel : la vocation naturelle de la femme n'est pas de se marier, mais d'enfanter, car «si une fille n'a pas d'enfants, elle devient hystérique ou malade $»^{46}$. Ce qui est vrai à l'échelle individuelle vaut aussi au niveau de la communauté dans son ensemble: «Ce qui a été voulu par la nature [n'est pas le] mariage [mais] la satisfaction de cette grande aspiration à la vie [qui consiste à ] imposer son droit à la vie ${ }^{47}$ ».

Dès lors, le pas vers le principe polygamique n'est plus long à franchir : préoccupé, voire terrifié par l'hémorragie que provoquent les combats, Hitler rappelle une fois encore le précédent du XVIIe siècle : « Après la guerre de Trente ans, la polygynie a été à nouveau autorisée : c'est par l'enfant illégitime que la nation s'est reconstituée ${ }^{48} »$.

$\mathrm{Au}$ plus haut niveau de la hiérarchie nazie s'acclimatent donc, dans un contexte de guerre, d'une guerre dure et coûteuse en hommes, des idées qui avaient été formulées dès les années 1920 dans les cercles les plus avant-gardistes du racisme völkisch et nazi, hostiles au mariage monogamique, institution suspecte, étrangère à la race germaniquenordique, et sans doute inventée pour assécher et tarir son flux reproductif.

Dans son maitre-ouvrage, La paysannerie comme source de vie de la race nordique (1929), Richard Darré affirmait ainsi que les Germains étaient polygames. Le rameau méridional, émigré en Grèce, de la race nordique, pratiquait une polygynie attestée par Plutarque qui rapporte qu'un "Spartiate pouvait demander à un autre de coucher avec son épouse », de même que «des hommes âgés pouvaient conduire leur jeune épouse vers un homme jeune pour lui demander un enfant, et ne considéraient pas cela comme honteux ${ }^{49}$ ». Il en va de même de la "Suède, patrie originelle de la race nordique, où l'on trouve des témoignages tardifs de polygynie ${ }^{50}$ ». Charles " le massacreur de Saxons », le Charlemagne évangélisateur et bourreau de la Saxe, était lui-même " polygame »: " le comportement de Charles le Massacreur de Saxons n'est en rien contraire à l'esprit nordique ${ }^{51}$ ", car les rois francs agissaient de même. Quant aux Romains, César « avait le projet d'introduire juridiquement la polygamie à Rome, afin de permettre une reproduction accrue ${ }^{52}$ » après le désastre démographique de la guerre civile.

61 Pour Darré, il est évident que l'institution sociale du mariage doit avoir pour fin unique la reproduction maximale et la prolifération de la race. Tout artéfact (social) qui contrevient à cette loi naturelle doit être aboli. Or, constate l'auteur, le mariage monogamique est devenu un dogme, car « la christianisation et l'acculturation romaine tardive ont modifié la culture juridique des Germains, ce qui a entraîné une évolution des concepts juridiques dans un sens antigermanique ${ }^{53}$ ».

62 Imposé à la race germanique avec et par le christianisme, le mariage monogamique est une institution néfaste à la vie de la race, une anti-nature solidaire des nombreux autres 
caractères d'une culture importée et hostile à la vie, comme en témoigne le rapport chrétien au corps : «Toute négation du corps a été, dès les origines, étrangère à la race nordique. C'est seulement au cours de l'antiquité, quand l'ombre monstrueuse d'une ascèse hostile à la beauté s'est levée de l'orient pour provoquer une éclipse de la culture que la déformation des concepts moraux a commencé et imposé l'idée d'un corps peccamineux ${ }^{54} \gg$.

Le christianisme comme anti-nature a aliéné la race en la dénaturant, en l'éloignant d'une conception saine et naturelle de la sexualité : le rapport sexuel de l'homme germanique doit être libre et servir la reproduction la plus libre et abondante de la race.

La dénonciation opiniâtre d'un christianisme qui a dénaturé la race en l'éloignant des principes, sains, de sa naissance et en l'empêchant d'obéir à ses principes élémentaires, est une des constantes des textes d'Alfred Rosenberg qui, lui aussi, et fort logiquement, prend parti pour la polygamie, non sans les concessions rhétoriques d'usage : «Certes, il faut protéger le mariage monogamique, mais [...] sans une polygamie temporaire, jamais les migrations germaniques des siècles passés n'auraient pu se dérouler et, donc, jamais la culture occidentale n'aurait pu éclore ${ }^{55}$ ». Le contexte démographique hérité de la Grande guerre rend cette question à nouveau actuelle : «Est-ce que ces millions de femmes, que l'on appelle avec commisération de 'vieilles filles', privées de leur droit à la vie, doivent traîner leur pauvre existence ainsi ? ». N’en déplaise aux clercs de tout poil, qui bénissent les mariages entre juifs convertis et chrétiens, c'est bien «la préservation d'une substance menacée de mort qui est l'essentiel, auquel doit être subordonné - et ceci exige la production d'un bon et sain sang allemand ${ }^{56}$ ». Pour clore ce passage intitulé « mariage biologique et mariage chrétien ", Rosenberg souligne que «les critères valides en temps de paix peuvent devenir dangereux et conduire à la catastrophe dans des temps marqués par un combat à la vie et à la mort. Le Reich allemand à venir devra évaluer (bewerten) toute cette question d'un nouveau point de vue et créer des formes de vie adéquates ${ }^{57}$ ».

C'est également, on s'en doute, la position de l'antichrétien convaincu Heinrich Himmler, qui attribue à l'aliénation chrétienne une grande partie des maux qui frappent la race germanique. Pour lui, le mariage monogamique est «une œuvre satanique de l'Église catholique $^{58}$ ", un instrument pour affaiblir la race en l'asséchant. Rien d'étonnant, quand on sait que le christianisme est une création des juifs pour détruire une race aryenne qu'ils ne parviennent pas à vaincre en combat ouvert ${ }^{59}$. Les juifs eux-mêmes savent bien que le combat biologique se gagne dans la bataille de la natalité : « Notre peuple vaincra s'il dispose d'assez de sang nordique, si ce sang prolifère, ou bien tombera s'il s'assèche. Dans ce cas, ce sera la fin de notre race et de sa culture. ${ }^{60}$ ”

Ce que pense et défend le Reichsführer SS est répercuté par les publications de son organisation. Ainsi de cet article du SS-Leitheft qui, en 1944, dans un contexte militaire et démographique épouvantable, rappelle que «la guerre totale est menée [...] avec des femmes et des enfants ${ }^{61}$ ». Dans ces conditions, «il en va de la vie ou de la mort de notre peuple et de notre race. Le caractère total de notre combat nous conduit à une radicale révolution de nos conceptions ». Foin du mariage et de l'obligation d'une reproduction conjugale, d'autant plus que les hommes manquent. De même que les hommes sont soumis au "devoir de combattre", de même les femmes ont-elles "le devoir de se reproduire »: «le combat biologique mené par notre peuple commandera de dépasser des conceptions de l'honneur féminin par trop prudes ». Les femmes qui se déroberont à ce qui est non plus une faute ou un manquement, mais un devoir biologique « devront être évaluées du point de vue social et moral comme elles le méritent. Elles n'ont pas plus 
de valeur que le planqué ou, au pire, le soldat déserteur. La guerre totale va donc aussi mener à une révolution contre les concepts moraux hypocrites de l'âge bourgeois ${ }^{62}$ ».

En pointe, cette promotion de la polygamie se retrouve très tôt chez les juristes qui exposent les projets de réforme du droit portés par le NSDAP. Ainsi de Helmut Nicolai, avocat et docteur en droit, qui publie, dans la maison d'édition du parti nazi sa Doctrine du droit racial. Principes d'une philosophie du droit national-socialiste (1932). A ses yeux, la régulation de la sexualité par une union sanctionnée par la communauté n'a pour seule fin, outre la multiplication des naissances, que le contrôle de la lignée. Si les femmes sont astreintes à la fidélité, c'est parce qu'il faut savoir si l'enfant né est bien de bonne race. Dès lors, il est logique que «le droit [germanique] ne connaissait pas l'adultère de l'homme quand il prenait une esclave auprès de lui, mais seulement l'adultère féminin [...] car si les femmes adultères deviennent la règle, nul ne peut plus savoir de qui sont les enfants ${ }^{63}$ ».

La justification de la polygynie figure donc en toutes lettres dans la littérature juridique du parti nazi dès 1932. Ces écrits militants reçoivent le soutien de la littérature scientifique: le plus célèbre historien du droit germanique de l'entre-deux-guerres, Claudius von Schwerin, consacre en 1938 un article au "Problème du mariage germanique » dans les colonnes de la Zeitschrift der Akademie für deutsches Recht ${ }^{64}$. Le savant commence par remarquer que l'opposition entre mono- et polygamie est anachronique, car les deux réalités coexistaient avant la victoire finale du christianisme et de sa " doctrine morale ${ }^{65}$ ", celle-là même qui dicte les jugements axiologiques que l'on entend porter sur les mœurs germaniques.

Pour Schwerin, il est évident que la polygamie était une pratique germanique répandue et approuvée, sous la forme plus précisément d'une polygynie masculine: cette pratique visait non pas à rabaisser la femme et à blesser des individus, mais à servir la race en permettant la reproduction du nombre maximal d'enfants. La relation asymétrique de la polygynie ne peut être appréhendée et évaluée dans les termes individualistes et jusnaturalistes de la culture contemporaine dominante, mais doit être évaluée dans les termes holistiques qui étaient ceux de la communauté germanique :

70 «Quiconque considère le mariage du point de vue de la race comprendra tout de suite [...] qu'il n'y a là aucun mépris de la femme, mais sa naturelle subsomption sous les intérêts de la race ${ }^{66}$ ». En effet, poursuit l'auteur, il ne faut pas voir « dans le mariage une simple relation exclusive entre époux, mais une communauté de vie et de destin qui [...] doit être considérée non du point de vue des époux, mais du point de vue du foyer et de la race ${ }^{67}$ ».

71 Le mariage n'est pas un contrat entre individus, mais l'institution qui perpétue une communauté raciale : « le mariage n'est pas formé pour les époux, mais pour la race, pour le développement de la race $^{68} "$.

72 La conception chrétienne d'un mariage comme sacrement est évacuée : ce qui est sacré, c'est la vie de la race et la prolifération de ses membres. Toute institution artificielle qui entrave cette fin naturelle doit être abolie.

73 Ces positions, très en pointe, ne font pas l'unanimité dans la corporation des juristes. Responsable, parmi d'autres, des travaux préparatoires à une refonte du Code civil, le professeur de droit Justus Hedemann précise en 1941 que " personne ne veut attenter à la monogamie [...] sous prétexte que la polygamie permet une natalité supérieure ${ }^{69} \ggg$, tout en reconnaissant que ces idées existent et sont débattues, et que si le mariage doit être 
protégé par le droit, il est subordonné à une fin qui lui est extérieure, et qui est « la fin supérieure de la multiplication et de la préservation de la race ${ }^{70}$ ».

D'autres publications témoignent des résistances culturelles à toute remise en cause du mariage monogamique, arguant que, pour lutter contre la dénatalité, il faut défendre la famille comme "cellule fondamentale" de l'organisation raciale. Ces publications, cependant, ne développent qu'une argumentation trop éculée pour être efficace, bêtement dogmatique et non dynamique: il faut selon eux revenir à un foyer monogamique et familial de 4 ou 5 enfants pour perpétuer la race et compenser les pertes de la guerre... Rien de bien offensif qui puisse convaincre et enthousiasmer les hiérarques du régime. En revanche, la critique de la monogamie est séduisante :

- Elle combat les présupposés chrétiens, issus d'une culture étrangère et néfaste, d'une normativité élaborée par des juifs et inoculée, comme un poison, à l'humanité germanique.

- Elle a le bénéfice de la rupture révolutionnaire avec des normes bourgeoises obsolète.

- Elle est révolution au sens propre du terme, sens repris et affectionné par les producteurs du discours nazi : rompant avec 1500 ans d'aliénation chrétienne, elle revient à la prime inspiration d'une race germanique qui connaissait la nature et ses lois, et savait agir en conséquence.

$\mathrm{Au}$ sein de la hiérarchie nazie, on l'a vu, la sexualité hors mariage est, à la condition qu'elle serve une fin reproductive, encouragée. Devant les pertes en hommes d'une guerre qui s'éternise, on réfléchit désormais, au-delà de la tolérance de fait, à une légalisation de la polygamie.

Les critiques et les voix divergentes sont réduites au silence. Le grand raciologue Hans Günther lui-même, héraut du racisme nordiciste le plus exigeant, est contraint de se taire par le NSDAP qui interdit en 1944 la parution de son essai sur Les enfants illégitimes considérés d'un point de vue racial ${ }^{11}$. Günther se proposait de défendre la monogamie comme gage de la stabilité des caractères héréditaires et comme garantie contre tout mélange et déperdition dans la ventilation des gènes. En un mot: un mariage monogamique et exclusif entre deux individus de bonne race est à ses yeux le moyen d'assurer une reproduction optimale du point de vue quantitatif (pour peu que le couple fasse un effort), mais aussi et surtout qualitatif, position qu'il défendait déjà dans son essai de 1941 sur Le choix des époux : condition du bonheur conjugal et de l'amélioration héréditaire ${ }^{72}$. Face aux assauts contre la monogamie, Günther veut réagir mais, en 1944, il ne le peut plus, car il est réduit au silence comme Fritz Lenz l'avait été en 1937 : aucune divergence ou critique n'est plus tolérée sur ce sujet.

77 La hiérarchie nazie veille, car il y a urgence, une urgence telle que la polygamie reproductive est pratiquée dans le cercle le plus rapproché autour d'Hitler. Le Reichsführer SS Heinrich Himmler possède deux foyers: le premier, issu d'un mariage qu'il juge précoce avec une femme qu'il estime trop âgée - ce dont il rend le catholicisme bavarois responsable ${ }^{73}$ - coexiste avec la relation quasi-officielle qu'il entretient avec son ancienne secrétaire, logée dans des bâtiments de la SS à Hohenlychen. Les visites dominicales d'Himmler à sa maîtresse et à ses deux enfants naturels, quoique discrètes, ne sont un mystère pour personne.

De même, la polygamie revendiquée de Martin Bormann, secrétaire particulier d'Hitler, dont le pouvoir, issu de la proximité au chef, croît considérablement entre 1940 et 1945 . Si on ignore ce que Mme Himmler pensait de la double vie de son mari, on sait par contre très bien que Gerda Bormann encourageait son mari à procréer en-dehors du cadre 
conjugal. Leur correspondance des mois de janvier et février 1944 montre bien que la relation extraconjugale est considérée, au plus haut sommet de la hiérarchie nazie, comme la mise en pratique d'un racisme nataliste conséquent. Martin et Gerda Bormann se donnent, dans leurs lettres, du « maman » et du «papa » : s'agit-il de l'usage bourgeois d'un vieux couple gâtifié par une trop longue vie commune et des naissances à répétition? Ou plutôt du signe qu'ils se considèrent avant tout comme des reproducteurs au service du Führer et du Reich?

79 À son mari, qui lui annonce sa liaison avec l'actrice Manja Behrens, Gerda Bormann répond: «C'est une pitié que de belles jeunes femmes comme ça se voient refuser d'être mères ${ }^{74}$ ", par une guerre qui les prive de leurs fiancés. Il est donc heureux que Martin se charge de l'engrosser: «Dans le cas de M., tu seras en mesure de changer ça, mais il faudra que tu veilles à ce que M. ait un enfant une année, et moi l'année suivante, de telle sorte que tu aies toujours une femme qui soit disponible ${ }^{75}$ ». Gerda Bormann organise un plan reproductif pluriannuel qui vise à la production maximal d'enfants par l'usage en alternance du ventre féminin «disponible». Elle sait cependant que Manja Behrens appartient encore au monde ancien. Eduquée chrétiennement, la maîtresse de son mari aura sans doute du mal à se plier à cette logique de reproduction polygamique bisannuelle. Il convient donc de l'initier à des idées moins néfastes à la race que ce christianisme: "Donne-lui les bons livres, mais fais-le discrètement, pour qu'elle en vienne aux justes conclusions à son rythme ${ }^{76}$ ».

80 Soucieuse de pédagogie et d'acculturation, Gerda Bormann se préoccupe également de législation. Le contexte démographique est tel qu'il faut agir, écrit-elle, sur le plan de la loi : "Il y a si peu d'hommes valables qui vont survivre à cette guerre que ça en est effrayant: tellement de femmes sont condamnées à rester seules parce que leur compagnon aura été tué dans une bataille - est-ce une fatalité ? Nous avons aussi besoin des enfants de ces femmes $!^{77}$ ». La saignée démographique des deux guerres mondiales rappelle - une fois encore - celle du XVIIe siècle. Dès lors, « il serait bon qu'une loi soit promulguée à la fin de cette guerre, comme à la fin de la guerre de Trente ans, qui permettrait à des hommes sains et valables d'avoir deux épouses ${ }^{78}$ ". La glose marginale de Martin Bormann précise : « Le Führer est exactement du même avis ${ }^{79}$ ».

81 Très en verve normative, Gerda Bormann forge le concept de "Volksnotehe ", expression que l'on pourrait traduire par «mariage d'exception raciale » ou "union de détresse raciale ». On retrouve dans ses propos l'écho des débats des années 1930 sur le statut de l'enfant illégitime et de la fille-mère. Soucieuse de protéger les femmes, Gerda Bormann précise que les nouvelles mesures législatives ou réglementaires ne devraient pas être « un prétexte pour des hommes peu scrupuleux qui y verraient l'occasion d'avoir des relations sexuelles partout, au prétexte d'avoir des enfants ${ }^{80}$ ». Pour éviter cela, «en aucun cas, les mères de ces enfants ne devraient avoir un statut inférieur à celui des femmes légalement mariées ${ }^{81} »$. Une fois ces précautions prises, il n'y a pas de limite à la multiplication des unions : "Chaque homme sain de corps et d'esprit, et membre valable de la communauté du peuple, devraient pouvoir contracter une union supplémentaire, ou plus $^{82}$ ». Gerda Bormann développe ses idées sur la garde alternée, la vie commune et la pension alimentaire. Tout est prévu, jusqu'au texte d'un formulaire à remplir pour contracter de telles unions : " Moi..., déclare que, avec le consentement de mon épouse..., je souhaite contracter une Volksnotehe avec... Cette union aura la même valeur devant la loi que mon premier mariage ${ }^{83} »$. 

n'est que l'écho des idées agitées par le Führer au même moment. Dans un memorandum daté du 29 janvier 1944 et intitulé « Assurer l'avenir de notre peuple ${ }^{85}$ », Martin Bormann note que « dans la nuit du 27 au 28 janvier 1944, le Führer s'est entretenu avec nous du problème de l'avenir de notre race ». Selon Hitler, « la situation de notre race sera, après la guerre catastrophique, car notre peuple affronte une seconde hémorragie terrible en moins de trente ans. Nous allons très certainement gagner la guerre, mais nous la perdrons du point de vue racial si nous ne révolutionnons pas nos conceptions héritées et les attitudes qui en découlent». Comme souvent Hitler rappelle les conséquences démographiques et contre-sélectives de toute guerre, avançant l'oliganthropie consécutive aux guerres de 1618-1648: «La guerre de Trente ans nous montre quelles conséquences politiques terribles une guerre peut avoir. Au début, le peuple allemand comptait 18 millions de personnes, à la fin, à peine 3 millions et demi. Les suites de cette hémorragie ne sont toujours pas compensées aujourd'hui, car nous y avons perdu la domination mondiale à laquelle, au début de la guerre de Trente ans, le peuple allemand était appelé ». Dans le contexte stratégique si inquiétant pour l'Allemagne nazie de l'hiver 1944, un an après Stalingrad, Hitler exagère à des proportions proprement fantastiques la saignée déjà si abondante de la guerre de Trente ans: les historiens estiment - et estimaient déjà - les pertes de population à $50 \%$, et non à $85 \%$ comme le fait ici le Führer ${ }^{86}$. Il reste que le déséquilibre démographique entre hommes et femmes sera, aux dires du Führer, catastrophique après la guerre: «trois ou quatre millions de femmes, qui n'auront jamais d'hommes!». Or ces femmes ne « concevront pas d'enfants sous la seule action du saint Esprit, mais bel et bien grâce aux hommes qui resteront ».

Il faudrait donc non seulement créer les cadres normatifs permettant cette reproduction d'urgence, mais aussi veiller à éclairer les esprits: «Dans ce domaine si délicat, les oukases de l'État sont impuissantes à elles seules [...]. Un travail de pédagogie est indispensable ». Il faudrait «donner des instructions précises aux écrivains et artistes contemporains : il faudra interdire les romans, nouvelles ou pièces de théâtre qui mettent en scène des drames conjugaux [...] ou les films, qui traitent l'enfant extraconjugal en enfant de valeur inférieure, en enfant illégitime ». Il en va de même des "prêtres ou pasteurs [...] qui prendront la parole contre ces nécessités raciales et qui seront sévèrement punis ».

L'impératif quantitatif, rendu si pressant par l'hémorragie démographique de la guerre, ne doit pas faire oublier la fin eugénique de la reproduction: le droit à la polygamie reproductive ne devra être accordé qu'aux hommes « dignes, forts, sains physiquement et psychologiquement »- en l'espèce, aux meilleurs soldats, aux héros de guerre. Un an avant ce monologue du Führer, Himmler confie à des proches qu'Hitler songe à accorder le privilège de polygamie aux plus haut décorés seulement ${ }^{87}$, ce qui montre que la préoccupation démographique (quantitative) reste indissociable du projet eugéniste (qualitatif). En janvier 1944 cependant, sans doute en raison de la dégradation continue de la situation militaire, ces restrictions sont oubliées: si, au printemps 1943, Hitler n'admettait au privilège polygame que les titulaires de la croix de fer de première classe, il n'est désormais plus question que d'un vague critère de santé et de bonne forme physique...

Dans un contexte de grande angoisse démographique léguée par les pertes inédites de la Grande Guerre, encore aggravé par celles de la Seconde Guerre mondiale, idéologues et hiérarques nazis en sont venus à imaginer une révolution des normes régissant la 
procréation d'enfants. Dans la mesure où l'impératif est la création de substance biologique de bonne race la plus abondante possible, tout obstacle à cette fin doit être levé : une institution sociale comme le mariage n'est qu'un artefact culturel qui ne peut entrer en contradiction avec les décrets naturels - d'autant plus, ou d'autant moins que cette institution monogamique trouve son origine dans les coutumes et commandements vétéro-testamentaires d'un peuple du désert dont la culture est venu dénaturer la race germanique.

Révolutionnaire au sens propre - et prérévolutionnaire du terme - la réflexion normative nazie veut retrouver la nature et la naissance de la race, enfouie sous les sédiments de siècles d'acculturation-dénaturation judéo-chrétienne. De même que le «tu ne tueras point » n'est pas une loi de Dieu, mais une loi des juifs, le mariage est l'objet d'une critique culturelle encore accentuée par un contexte démographique de plus en plus préoccupant au fil des défaites militaires nazies.

Pour permettre une reconstitution de la substance raciale après la guerre, c'est bel et bien la nature, et non l'Église, les Dix commandements, le droit civil ou les préjugés de la morale victorienne, qui devra légiférer : en matière de procréation, comme de combat ou de meurtre, les nazis, fort conséquemment, consacrent la biologie - ou, du moins, ce qu'ils entendent par biologie ou Naturgesetzlichkeit - comme seule loi.

\section{NOTES}

1. Otto Helmut(dir.), Volk in Gefahr. Der Geburtenrückgang und seine Folgen für Deustchlands Zukunft, München : Lehmann, 1934, $59 \mathrm{p}$.

2. Ibid., p. 35.

3. Ibid., p. 21.

4. Ibid., p. 6.

5. Ibid., p. 52.

6. Ibid., p. 54.

7. Ibid., p. 53.

8. Ibid., p. 53.

9. Ibid., p. 57.

10. Friedrich Burgdörfer, Völker am Abgrund, Politische Biologie - Schriften für naturgesetzliche Politik und Wissenschaft - Heft 1, München : Lehmanns Verlag, 1936, 60 p.

11. cf. Johann Chapoutot, Le national-socialisme et l'antiquité, Paris : PUF, 2008, 532 p.

12. Le terme allemand pour désigner l'enfant «naturel » ou "illégitime » est " ausserehelich » soit, littéralement, « extraconjugal ». L'emploi du terme « naturel » - français et non allemand nous permet ici un jeu de mots qui exprime bien cet argument nazi selon lequel l'enfant « naturel » est un don de la nature, à valoriser et à chérir. Ce qui est « illégitime » n'est pas l'enfant « naturel », mais bien toute condamnation sociale ou morale de son avènement.

13. Richard Walther Darré, Neuadel aus Blut und Boden, München : Lehmann Verlag, 1930, p. 172.

14. Richard Walther Darré, Neuordnung unseres Denkens, Reichsbauernstadt Goslar : Verlag Blut und Boden, Die Goslarer Volksbücherei, 1940, p. 36.

15. Ibid., p. 43 
16. R. Haff, « Der Sippengedanke im deutschen Recht » in : Deutsches Recht, 1935, p. 84-86, p. 85.

17. Ibid.

18. Ibid.

19. «Das Schwarze Korps », 28 octobre 1937, cité in: Josef Ackermann, Heinrich Himmler als Ideologe, Göttingen : Musterschmidt, 1970, p. 128.

20. Kurt Schmidt-Klevenow, « Das uneheliche Kind in der Volksgemeinschaft », Deutsches Recht, 1937, p. 148-152.

21. Ibid., p. 150.

22. Friedrich Lenz, « Zur Frage der unehelichen Kinder », Volk und Rasse, mars 1937, p. 91-95.

23. Ibid., p. 93.

24. Josef Ackermann, Heinrich Himmler als Ideologe, op. cit., p. 130.

25. Kurt Schmidt-Klevenow, « Das uneheliche Kind... », article cité, p. 151.

26. Onze établissements en 1945, dont huit hospices pour les mères et trois hospices d'enfants sous tutelle de la SS. En tout, ce sont 11000 enfants qui sont nés dans les Lebensborn entre 1936 et 1945. Cf. Ackermann, p. 129.

27. Heinrich Himmler, cité in Volker Koop, Dem Führer ein Kind schenken - Die SS-Organisation Lebensborn e.V., Köln : Böhlau, 2007, p. 42.

28. Service, tâche, ou mission. Le mot allemand employé ici est Dienst.

29. Franz Wieacker, « Geschichtliche Ausgangspunkte der Ehereform » in : Deutsches Recht, 1937, p. $178-184$.

30. Ibid., p. 179.

31. Karl Larenz, "Grundsätzliches zum Ehescheidungsrecht » in: Deutsches Recht, 1937, p. 184-188.

32. Ferdinand Mössmer, «Der Gemeinschaftsgedanke im Recht der Eheschliessung und Ehescheidung » in : Deutsches Recht, 1935, p. 86-88.

33. Ibid., p. 86.

34. Ibid., p. 86.

35. Ibid., p. 87.

36. Ibid., p. 87.

37. Ibid., p. 87.

38. Rudolf Hess, «Der höchste Dienst der Frau für Deutschland - Rudolf Hess an eine unverheiratete Mutter », Völkischer Beobachter, 24-26 décembre 1939, p. 11.

39. Ibid.

40. Der Führer an die Deutschen Frauen, 8 septembre 1934, Nuremberg.

41. Bund Deutscher Mädel (Association des jeunes filles nazies), Winterhilfswerk (Æuvre du secours d'hiver) et Nationalsozialistische Volkswohlfahrt (Association d'entraide nationale-socialiste).

42. Hitler, Adolf, propos privés du 23 avril 1942, midi, Wolfschanze in : Henry Picker (éd.), Hitlers Tischgespräche im Führerhauptquartier : 1941-42, Bonn : Athenäum, 1951, 463 p., rééd. Stuttgart : Seewald, 1976, p. 235.

43. Hitler, Adolf, propos privés du 15 mai 1942, in : Henry Picker (éd.), Hitlers Tischgespräche im Führerhauptquartier, op. cit., p. 289.

44. Hitler, Adolf, propos privés du 14 avril 1942, in : Henry Picker, op. cit., p. 297.

45. Comme ce fut le cas de Reinhard Heydrich, renvoyé de la marine pour avoir rompu ses fiançailles. Cf. Robert Gerwarth, Reinhard Heydrich. Biographie, München : Siedler, 2011, 478 p., pp. 52-59.

46. Hitler, Adolf, propos privés du $1^{\mathrm{er}}$ mars 1942, in : Henry Picker, op. cit., p. 118.

47. Ibid., p. 118.

48. Ibid., p. 117.

49. Richard Walther Darré, Das Bauerntum als Lebensquell der nordischen Rasse, München: Lehmanns Verlag, 1929, $8^{\text {ème }}$ rééd. 1940, p. 351. 
50. Ibid., p. 399.

51. Ibid.

52. Ibid., p. 400.

53. Richard Walther Darré, Neuadel aus Blut und Boden, op. cit., p. 133.

54. Richard Walther Darré, Das Bauerntum als Lebensquell, op. cit., p. 445

55. Rosenberg, Der Mythus des 20.Jahrhunderts, p. 594.

56. Ibid., p. 595.

57. Ibid.

58. Heinrich Himmler à son masseur Felix Kersten, cité in: Volker Koop, Dem Führer ein Kind schenken - Die SS-organisation Lebensborn e.V., Köln : Böhlau, 2007, p. 41.

59. Cf. Johann Chapoutot, Le national-socialisme et l'antiquité, op. cit.

60. Heinrich Himmler, discours du 2 septembre 1938, cité in: Volker Koop, Dem Führer ein Kind schenken, op. cit., p. 47.

61. Ludwig Eckstein, « Die biologische Seite des totalen Krieges », SS-Leitheft, 1944, Heft 2, p. 19-23.

62. Ibid., p. 21.

63. Helmut Nicolai, Die rassengesetzliche Rechtslehre. Grundzüge einer nationalsozialistischen Rechtsphilosophie, Nationalsozialistische Bibliothek, Heft 39, München : Franz Eher Verlag, 1932, p. 13.

64. Claudius Freiherr von Schwerin, «Zum Problem der germanischen Ehe » in : Zeitschrift der Akademie für deutsches Recht, Berlin/München : Beck, 1938, p. 529-532.

65. Ibid., p. 530.

66. Ibid., p. 531

67. Ibid., p. 532

68. Ibid., p. 531.

69. Justus Wilhelm Hedemann, Das Volksgesetzbuch der Deutschen. Ein Bericht, München : Beck, 1941 , p. 12.

70. Ibid., p. 30.

71. cf. Hans-Christian Harten, Uwe Neirich, Matthias Schwerendt (dir.), Rassenhygiene als Erziehungsideologie des Dritten Reiches, Akademie-Verlag, 2006, p. 143-144.

72. Hans Friedrich Karl Günther, Gattenwahl zu ehelichem Glück und erblicher Ertüchtigung, München : Lehmann, 1941, $171 \mathrm{p}$.

73. Peter Longerich, Heinrich Himmler. Biographie, Frankfurt : Pantheon, 2010, p. 116 et p. 377-378.

74. Hugh Trevor-Roper, (éd.), The Bormann Letters. The Private Correspondence between Martin Bormann and his Wife from January 1943 to April 1945, London : Weidenfeld and Nicolson, 1954, p. 41.

75. Ibid., p. 42.

76. Ibid., p. 43.

77. Ibid., p. 45 .

78. Ibid., p. 45.

79. Ibid., p. 45.

80. Ibid., p. 49

81. Ibid., p. 49.

82. Ibid., p. 50.

83. Ibid., p. 50.

84. "C'est quand même étrange, qu'un mari écrive à sa femme qu'il est amoureux d'une autre femme », Gerda à Martin Bormann, p. 46.

85. Jochen von Lang, Der Sekretär : Martin Bormann, der Mann, der Hitler beherrschte, Stuttgart, 1977, p. $478-82$.

86. Cf. Henry Bogdan, La guerre de Trente ans, Paris : Perrin, 1997, rééd. Tempus, 2006, p. 270-279. 
87. Conversation d'Heinrich Himmler avec Felix Kersten, 4 mai 1943. Citée in: Volker Koop, Dem Führer ein Kind schenken, op. cit., p. 41.

\section{AUTEUR}

\section{JOHANN CHAPOUTOT}

Johann Chapoutot est maître de conférences des Universités à l'université Pierre-Mendès-France et membre de l'Institut universitaire de France. 